\title{
Keynote: Trustworthy Services and the Biological Analogy
}

\author{
Michael Reiter \\ Department of Computer Science \\ University of North Carolina at Chapel Hill \\ Campus Box 3175, Sitterson Hall \\ Chapel Hill, North Carolina 27599-3175, USA \\ reiter@cs.unc.edu
}

\begin{abstract}
Biological systems survive through a combination of redundancy, diversity and modularity. It has been argued that these principles can also be applied to construct information services that survive a variety of hostile attacks, including even the compromise of computers that help implement the service. Despite nearly 30 years of research to advance these principles and to apply them to the construction of trustworthy services, each remains an active and fruitful topic of research. In this talk we will describe recent progress in achieving redundancy, diversity and modularity, and in using these to implement trustworthy services. This progress, we will argue, is paving the way to next-generation services that are significantly more resilient than todays. We will also discuss challenges that remain in achieving this goal.
\end{abstract}

